\title{
New analysis of square section column plastic folding under axial impact for offshore shock absorbers
}

\author{
A. Almasi \\ Department of Mechanical Engineering, Tecnicas Reunidas S.A., Spain
}

\begin{abstract}
In modern offshore installations, thin walled columns are used for energy absorption in accident situations. When this advanced offshore shock absorber is subjected to axial compressive load, plastic hinges are developed on the sides of column and the column crushes with repeatable patterns. It can absorb considerable impact energy due to large and continuous plastic deformation. This paper develops a new closed form, energy based, solution for the prediction of accurate collapse curves of plastic folding of square section columns under axial impact load. New formulations for square section tube plastic folding, with respect to contributions from hinge curvature, strain hardening, second force oscillation and sensitivity to imperfection, are presented. The behaviour of a column in dynamic load is investigated using a simplified model of an offshore impact with dynamic corrections, including strain rate modelling. Good agreement between experimental and theoretical load-displacement and velocitytime curves verifies the accuracy of the proposed formulation.
\end{abstract}

Keywords: offshore shock absorbers, collapse, axial impact, square section column.

\section{Introduction}

The design of offshore shock absorbers has great effect on the reliability of ships and offshore structures. When a relatively short thin-walled closed section column is subjected to an axial compressive load, horizontal and inclined yield lines are developed along the sides of the column and the column crushes with a repeatable pattern $[1,2]$. This type of crushing is known as "Plastic Folding". Fig.1 shows the plastic folding of a square tube [2]. The corresponding 
experimental load-deflection curve oscillates between an upper and a lower limit wherein the maximum axial force corresponds to the initiation of each cycle of plastic folding. Due to the large and continuous plastic deformation of the tube, it can absorb considerable energy. A major issue in the design of the offshore shock absorber is its energy absorbing capability so that during a crash, the integrity of the offshore structure is ensured and only minimum forces are applied to the systems. Various analytical and numerical simulation techniques have been proposed. Numerical techniques such as the finite elements method are highly sensitive to the topology and density of meshes, and require continual re-meshing of the structure. Furthermore, the results of these simulations cannot be readily generalized and hence do not support parametric studies. Analytical methods, on the other hand, use individual elements with explicit formulations derived from the kinematics of the structure. These methods systematically rely on careful observations of the actual plastic deformation process in their derivation of the relations and verification of their results.

In the 1980s a number of theories were postulated to predict the mean force of the plastic folding of square tubes. It was reported [3] that the majority of tubes crushed symmetrically. Also, for the first time, two asymmetric modes of square tube plastic folding (asymmetric modes ' $\mathrm{A}$ ' and ' $\mathrm{B}$ ') were identified. Researchers published their theoretical results and experimental observations about the crushing of tapered sheet metal tubes [4,5], foam-filled square tubes [6] and multi-corner sheet metal columns [7]. The magnitude of force oscillations can be as large as half of the average crushing load [8]. In a design situation, if such a structure is used to absorb energy, or to resist a minimum load over a large range of plastic deformations, it is necessary to estimate the forcedisplacement and the force-time behaviours of the shock absorber under dynamic conditions. Analytical formulation for the load-deformation of round stocky tubes in plastic folding was reported in [8]. It was based on "concentrated plastic hinge" and "rigid-plastic model" assumptions and predicted load much lower than experimental data.
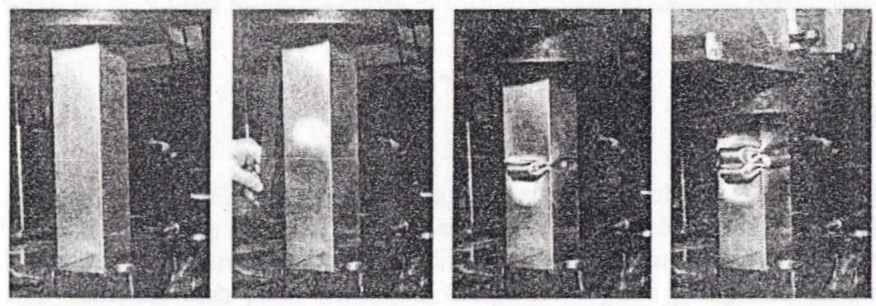

Figure 1: $\quad$ Plastic folding of square tube [2].

An alternative method for determining the behaviour of round stocky tubes was presented based on a model that included the plastic hinge curvature [9]. The formulation, however, was derived for two special cases, namely those of the plastic hinge curvature length being equal to one third and one half of the folding wavelength and as noted just for cylinders. 
In this paper a new analytical formulation for plastic folding of square section tube is presented which takes into account the effects of plastic hinge curvature, strain hardening, strain rate, second oscillation of force and sensitivity to imperfections. In addition a dynamic model for the prediction of the plastic folding behaviour is presented. Analytical results are compared with experimental data for both quasi-static and dynamic loadings.

\section{Modelling of folding mechanisms}

Experimental observations show four different mechanisms for the plastic folding of square tubes $[1,3]$. The first mechanism is the well-known symmetric mode which two opposite sides of each "layer" move inwards, and the other two move outwards. Each formed layer is oriented at 90 degrees to the previous one. The Symmetric mode is the most common folding mechanism for the crushing of square tubes. This mode is quasi-inextensional, as no extension of the area or the plastic hinge length occurs in the process except for a minor extension of the toroidal surface. In the practical geometrical range of thin-walled square tubes, the crushing of square tubes under axial impact is theoretically governed by symmetric mode [1,3]. Two asymmetric modes of deformation named "type A" and "type B" were also identified $[1,3]$. Both modes predict a similar symmetric mode for the first layer. Beyond the first layer, the asymmetric mixed mode "A" has a layer with three individual lobes deforming outwards and one deforming inwards. The asymmetric mixed mode ' $\mathrm{B}$ ' has two adjacent lobes deforming outwards with the other two adjacent lobes deforming inwards [1].

Based on previous observations, the plastic folding of thick wall square tubes is controlled by the "extensional mode" [3]. In "extensional mode", all four sides of each layer move outwards. A thorough discussion of the aforementioned plastic folding modes is included in [1,3]. Various known folding mechanisms can be modelled by mixing two basic folding elements, "quasi-inextensible" and "extensible". They are building blocks for various plastic folding mechanisms of square tubes. Characteristics of these two elements are elaborated in $[1,3,10]$. The Quasi-inextensible folding element has two types of plastic hinges. First type is horizontal (or fixed) plastic hinge, which appears in the upper and lower limits of the element as well as the midpoint between the two limits. The second type is inclined (or moving) plastic hinge. As the deformation process proceeds, one of the sides between the horizontal hinges folds inwards and the other folds outwards and a toroidal surface is thus developed. The extensional folding element has horizontal plastic hinges, which means that during the folding process the sides between hinges move outwards. The idealized symmetric collapse mode for a square tube consists of four quasi-inextensible elements $[1,3]$. The asymmetric mixed deformation modes ' $A$ ' and ' $B$ ' consists of six quasi-inextensible plus two extensible elements, and seven quasi-inextensible plus one extensible folding element, respectively [1]. Both asymmetric patterns consist of two folding layers. The extensional folding mode consists of one layer with four extensible elements. 
A second smaller peak load is observed in the majority of experimental loaddisplacement curves (two peak loads in each cycle of plastic folding). This phenomenon is attributed to the non-simultaneous proceeding of the upper and the lower folding sides [9]. Experimental curves show that the first load cycle is different from the rest of the deformation process and peaks at a higher value. The imperfections produced as a result of the initial elastic-plastic deformation of the first loading are depicted in [9]. Once the first load cycle is completed, subsequent load cycles start on a portion of the tube that already has an initial imperfection. Comparisons show that the analytical methods based on concentrated plastic hinges tend to underestimate the actual plastic folding load [8]. A significant part of the energy absorbed by the collapse mechanism cannot be modelled properly in the concentrated hinges approaches. Furthermore, detailed observations of the plastic folding mechanism shows that a larger region around the hinge is experiencing plastic deformation because the material in the immediate vicinity of the plastic hinge is strain-hardened and yielding shifts to an immediate neighbouring region. Second oscillation of force, initial imperfection, hinge curvature and strain hardening are modelled in this paper to represent realistic behaviour of force-displacement and force-time of plastic folding. Fig.2 shows model of a plastic folding including the plastic hinge curvature.

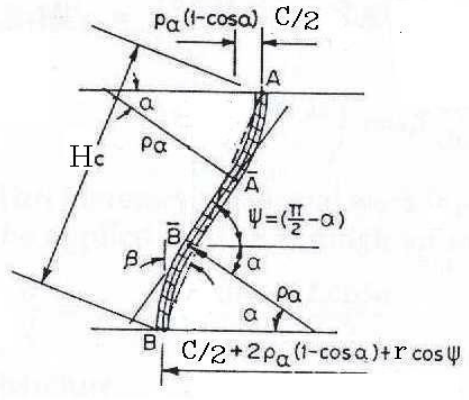

$A \bar{A}=\bar{A} \bar{B}=\bar{B} B=\rho_{\alpha} a=r$

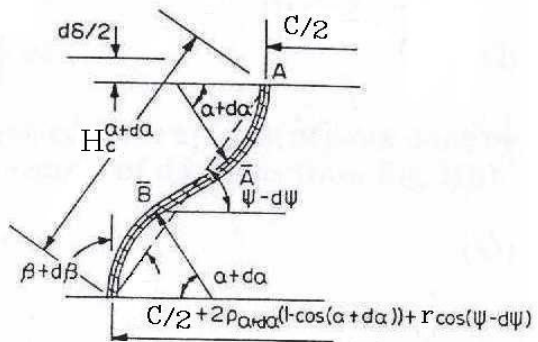

Figure 2: $\quad$ Model for plastic folding.

\section{Formulation of plastic folding collapse}

The folding wavelength $(\mathrm{H})$ and the hinge curvature length $(\mathrm{r})$ are both assumed to be constant. Based on formulations of quasi-inextensible and extensible elements (including horizontal and inclined hinges, toroidal surface and extension of the sides), the governing equation for the crush load of the symmetric plastic folding of a square tube is as eqn (1). The governing equations for the crush load in asymmetric mode ' $\mathrm{A}$ ' and mode ' $\mathrm{B}$ ' and the extensional mode can be obtained in the same manner. 


$$
\begin{aligned}
& \sigma_{f b 1}\left(1-\left(\frac{P}{P_{f b 1}}\right)^{2}\right) h c+\frac{\sigma_{f i}}{2}\left(\frac{1-\left(\frac{P}{P_{f i}}\right) \cos (\alpha)}{\sqrt{4-3\left(\frac{P}{P_{f i}}\right)^{2} \cos ^{2}(\alpha)}}\right) \frac{h H_{c}}{b} \xi\left(1+\sin ^{2}(\beta)\right)^{\frac{1}{2}} \frac{d \beta}{d \alpha} \\
& +2 \sigma_{f t}\left(-\left(\frac{P}{P_{f t}}\right) \cos (\alpha)+\sqrt{\left.4-3\left(\frac{P}{P_{f t}}\right)^{2} \cos ^{2}(\alpha)\right)} b \xi\left(\int_{0}^{\gamma} \frac{d \varphi}{\sqrt{1+\cos ^{2}(\varphi)}}\right) \frac{d \beta}{d \alpha}\right. \\
& -\frac{P}{2 h}\left[2 r \frac{\sin (\alpha)}{\alpha^{2}}-2 r \frac{\cos (\alpha)}{\alpha}+(H-2 r) \sin (\alpha)\right]=0
\end{aligned}
$$

where

$$
\begin{gathered}
\gamma=\tan ^{-1}(\sqrt{2} \tan (\beta)) \quad \beta=\cos ^{-1}\left(\frac{\xi}{H_{c}}\right) \quad \xi=2 r \frac{\sin (\alpha)}{\alpha}+(H-2 r) \cos (\alpha) \\
H_{c}=\sqrt{(H-2 r)^{2}+8 r^{2}\left(\frac{1-\cos (\alpha)}{\alpha^{2}}\right)+4 r(H-2 r) \frac{\sin (\alpha)}{\alpha}} \\
\left.\frac{d \beta}{d \alpha}=\frac{J M^{\prime}-2 M J^{\prime}}{2 M \sqrt{M-J^{2}}}\right) \\
J=2 r \frac{\sin (\alpha)}{\alpha}+(H-2 r) \cos (\alpha), M=(H-2 r)^{2}+8 r^{2} \frac{(1-\cos (\alpha))}{\alpha^{2}}+4 r(H-2 r) \frac{\sin (\alpha)}{\alpha} \\
J^{\prime}=2 r\left(\frac{\alpha \cos (\alpha)-\sin (\alpha)}{\alpha^{2}}\right)-(H-2 r) \sin (\alpha), M^{\prime}=16 r^{2} \frac{(1-\cos (\alpha))}{\alpha^{3}}+4 r(H-2 r)\left(\frac{\sin (\alpha)+\alpha \cos (\alpha)}{\alpha^{2}}\right)
\end{gathered}
$$

The relationship between the axial folding distance and the inclination angle of the folding side $(\alpha)$ can be written as eqn (2). The locking angle ( $\alpha$ f) of the folding mechanism is obtained by solving eqn (3). This angle determines the maximum deflection of the layer and defines the end point of each folding cycle. The angle lies within the range of 90 to 180 degrees.

$$
\begin{gathered}
\delta=2 H-4 r \frac{\sin (\alpha)}{\alpha}-(2 H-4 r) \cos (\alpha) \\
\frac{h}{2}+\frac{r}{\alpha}-2 r \frac{\sin (\alpha)}{\alpha}-(H-2 r) \cos (\alpha)=0
\end{gathered}
$$

Expressions for the mean effective strain are derived based on the principle of incompressibility. Different mean effective strain expressions are derived for the horizontal and inclined hinge plastic zones, boundary and middle horizontal hinge plastic zones, toroidal surface plastic zones, and extension of side plastic zones to incorporate strain effect in model. By integrating axial load over a cycle of plastic folding, and making the same simplification as $[1,3,10]$, the mean crushing load can be formulated and by minimizing this load with respect to folding parameters $(\mathrm{H}$ and $\mathrm{b})$, folding parameters are determined. The mean folding load, folding wavelength and radius of toroidal surface are represented by eqns (4), (5) and (6) respectively. 
230 Fluid Structure Interaction V

$$
\begin{aligned}
& \frac{P_{m}}{M_{p}}=k_{11}\left(\frac{c}{h}\right)^{\frac{1}{3}}, k_{11}=\left(I_{11} k_{13}+\frac{I_{12}}{k_{12}}+I_{13} \frac{k_{12}}{k_{13}}\right) \\
& \frac{H}{h}=k_{12}\left(\frac{c}{h}\right)^{\frac{2}{3}}, k_{12}=\left(\frac{I_{11}}{I_{13}}\right)\left(k_{13}\right)^{2} \\
& \frac{b}{h}=k_{13}\left(\frac{c}{h}\right)^{\frac{1}{3}}, k_{13}=\left(\frac{I_{12} I_{13}}{I_{12}^{2}}\right)^{\frac{1}{3}}
\end{aligned}
$$

where

$$
I_{11}=\frac{32 J_{11}}{J_{3}}, I_{12}=\frac{8 J_{13}}{J_{3}}, I_{13}=\frac{8 J_{12}}{J_{3}}
$$

Using a similar approach, the mean force and the folding parameters of asymmetric mode ' $A$ ' and ' $B$ ' and extensional folding mechanism can be determined. Three coefficients of these equations are presented below as eqns (7) to (9). Other coefficients can be obtained with a similar approach.

$$
\begin{aligned}
& J_{11}=\int_{0}^{\alpha_{f}} F_{f t}(\alpha) f_{2}(\alpha) f_{3}(\alpha) f_{5}(\alpha) d \alpha \\
& J_{12}=\int_{0}^{\alpha_{f}} F_{f i}(\alpha) f_{1}(\alpha) f_{2}(\alpha) f_{3}(\alpha) f_{4}(\alpha) d \alpha \\
& J_{13}=\int_{0}^{\alpha_{f}} F_{f b 1}(\alpha) d \alpha
\end{aligned}
$$

where

$$
\begin{gathered}
f_{1}(\alpha)=\sqrt{(1-2 k)^{2}+8 k^{2}\left(\frac{1-\cos (\alpha)}{\alpha^{2}}\right)+4 k(1-2 k) \frac{\sin (\alpha)}{\alpha}} \\
f_{2}(\alpha)=2 k \frac{\sin (\alpha)}{\alpha}+(1-2 k) \cos (\alpha) \quad f_{3}(\alpha)=\frac{d \beta}{d \alpha} \\
f_{4}(\alpha)=\left(1+\sin ^{2}(\beta)\right)^{\frac{1}{2}}, \beta=\cos ^{-1}\left(\frac{f_{2}(\alpha)}{f_{1}(\alpha)}\right) \\
f_{5}(\alpha)=\int_{0}^{\alpha_{f}}\left(\int_{0}^{\gamma} \frac{d \phi}{\sqrt{1+\cos ^{2}(\phi)}}\right) d \alpha, \gamma=\tan ^{-1}(\sqrt{2} \tan (\beta)) \\
f_{6}(\alpha)=2 k\left(\frac{1-\cos (\alpha)}{\alpha}\right)+(1-2 k) \sin (\alpha) \\
f_{7}(\alpha)=-\left(2 k \frac{\sin (\alpha)}{\alpha^{2}}-2 k \frac{\cos (\alpha)}{\alpha}+(1-2 k) \sin (\alpha)\right)
\end{gathered}
$$

Factors 'Fft', 'Ffi' and 'Ffb1' are strain rate factor expressions. 


\section{Dynamic effects}

In an offshore accident, the kinetic energy of the ship is transformed to the plastic deformation of shock absorber members. Elastic-plastic behaviour governs the initial loading of plastic folding [11-13] as well as the transition from minimum load to maximum load in each load cycle. The equation of motion can be expressed as (10).

$$
-F[x(t)]=M \frac{d^{2} x(t)}{d t^{2}}
$$

To introduce the strain rate effects to the model, the empirical Cowper-Symons uni-axial constitutive equation as eqn $(11)$ is used $[1,3]$.

$$
\sigma^{\prime}=\sigma\left(1+\left(\frac{\dot{\varepsilon}(t)}{q}\right)^{\frac{1}{p}}\right)
$$

\section{Comparison with experimental results}

Fig. 3 shows the experimental load-displacement curve from [1] being compared with three theoretical curves. The experimental curve belongs to the quasi-static symmetric crushing of a square section steel tube [1]. The solid curves are plotted according to eqn (1). The dash-dotted curve represents the loaddisplacement behaviour without the strain hardening effect and the dashed curve represents the same behaviour without the hinge curvature and strain hardening effects. Fig. 3 shows how each refinement of the analysis brings the solution closer to the actual collapse curve of the column.

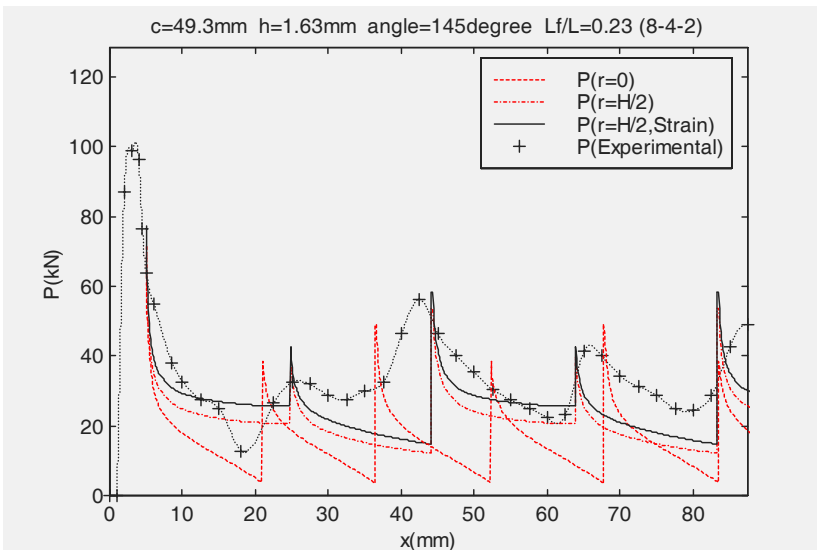

Figure 3: Load-displacement curves.

Experimental and theoretical velocity-time curves are plotted in Fig. 4. The theoretical curve represents the velocity-time behaviour of the dynamic model considering the plastic hinge curvature, strain hardening, strain rate, second oscillation of the force and sensitivity to imperfections. There is good agreement 
between theoretical results and experimental data. Fig. 5 shows the theoretical load-time curves corresponding to Fig. 4. A decrease in the velocity would cause the time of each plastic folding cycle to increase. Force peaks are more than yielding force due to strain rate and strain effects.

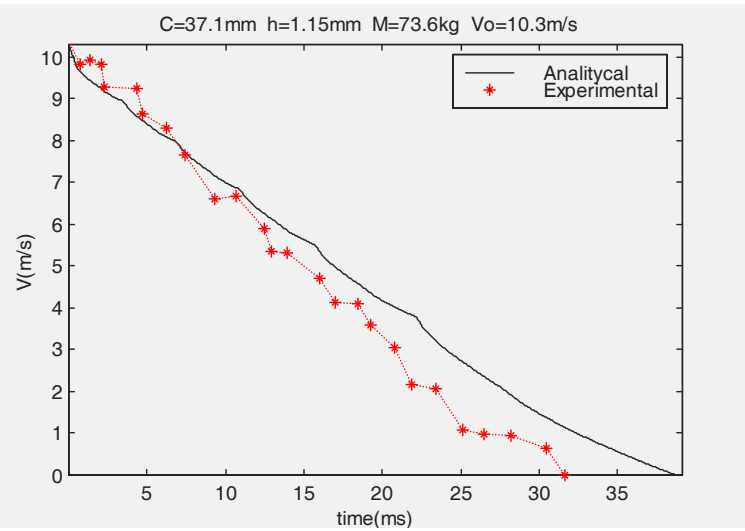

Figure 4: Velocity-time curve

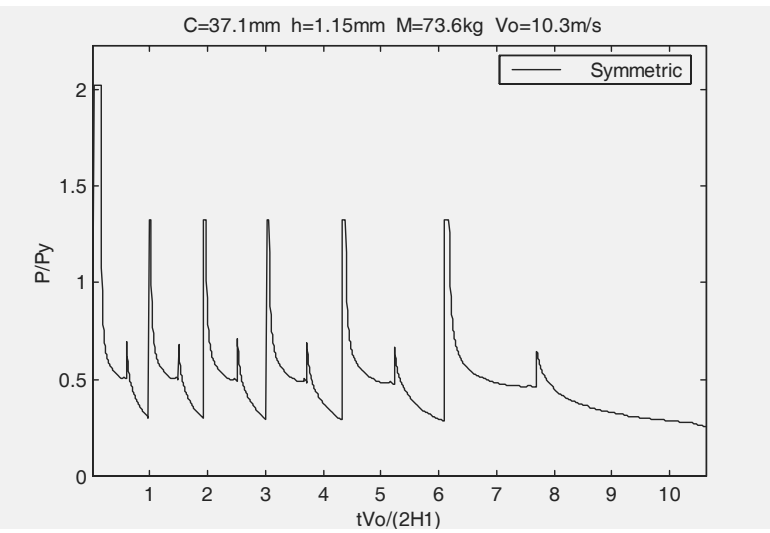

Figure 5: Theoretical load-time curve.

\section{Summary and conclusions}

New method for analysis of plastic folding of square section column is presented. Collapse of square section tube is formulated taking into account contributions from hinge curvature, strain hardening, strain rate, second force oscillation and sensitivity to imperfections. The dynamic behaviour of a square tube in plastic folding under axial impact is investigated using a simplified dynamic model of an offshore accident. Good agreement between experimental and theoretical load-displacement and velocity-time curves verifies the accuracy of the proposed formulation. 


\section{References}

[1] W. Abramowitz and N. Jones, Dynamic Axial Crushing of Square Tubes, Int. J. of Impact Engage, Vol. 2, pp. 179-208, 1984.

[2] M. Lanseth and O. S. Hopers tad, Local Buckling of Square Thin-Walled Aluminium Extrusions, J. of Thin-Walled Structures, Vol. 27, No. 1, pp. 117-126, 1997.

[3] W. Abramowitz and N. Jones, Dynamic Progressive Buckling of Circular and Square Tubes, Int. J. Impact Engage, Vol. 4, No. 4, pp. 243-270, 1986.

[4] S. R. Reid, T. Y. Reddy, Static and Dynamic Crushing of Tapered Sheet Metal Tubes of Rectangular Cross-Section, Int. J. Mech. Sci., Vol. 28, No. 9, pp. 623-637, 1986.

[5] A. G. Mamalis, D. E. Melonakos, G. L. Vigilant, The Axial Crushing of Thin PVC Tubes and Frusta of Square Cross-Section, Int. J. Impact Engg., Vol. 8, No. 3, pp. 241-264, 1989.

[6] W. Abramowitz and T. Wierzbicki, Axial Crushing of Foam Filled Columns, Int. J. Mech. Sci., Vol. 30, No.3/4, pp. 263-271, 1988.

[7] W. Abramowitz, T. Wierzbicki, Axial Crushing of Multicorner Sheet Metal Columns, J. Applied Mech., Vol. 56, pp. 113-120, 1989.

[8] R. H. Grzebieta and N. W. Murray, Rigid-Plastic Collapse Behaviour of an Axially Crushed Stocky Tubes, Recent Advances in Impact Dynamics of Engineering Structures, 1989.

[9] R. H. Grzbieta, An Alternative Method for Determining the Behaviour of Round Stocky Tubes Subjected to an Axial Crush Load, Thin walled Structures, Vol. 9, pp. 61-89,1990.

[10] W. Abramowicz, The Macro Element Approach in Crash Calculations, Proceeding of the Natal-ASI on Crashworthness of Transportation Systems Structural Impact and Occupant Protection, July 7-19, Troia, Portugal, 1996.

[11] Paulius Griskevicuis, Antanas Ziliukas, The Crash Energy Absorption of The Vehicle Front Structures, Transport, Vol XVIII, No2, 97-101, 2003.

[12] C.B.W. Pedersen, Topology Optimization of Energy Absorbing Frames, Fifth World Congress on Computational Mechanics, Vienna, Austria, July 7-12, 2002.

[13] Denzil G. Vaughn, James M. Canning, John W. Hutchinson, Coupled Plastic Wave Propagation and Column Buckling, Journal of Applied Mechanics, Vol. 72, January 2005. 